\title{
APLICAÇÃO PARA A WEB PARA ANÁLISE DE IMAGENS TÉRMICAS EM AGRICULTURA DE REGADIO
}

\author{
Tiago Levita ${ }^{1}$, Diana Gonçalves ${ }^{2}$, Qingfeng Miao ${ }^{3}$ e José Manuel Gonçalves ${ }^{4}$ \\ ${ }^{1}$ Software Engineer, Coimbra, Portugal \\ ${ }^{2}$ Biomedical Engineer, Coimbra, Portugal \\ ${ }^{3}$ College of Water Conservancy and Civil Engineering of Inner Mongolia Agricultural University \\ Hohhot, China \\ ${ }^{4}$ Escola Superior Agrária, Politécnico de Coimbra, Linking Landscape, Environment, Agriculture and Food \\ Coimbra, Portugal
}

\begin{abstract}
RESUMO
As técnicas de detecção remota para monitorização das culturas agrícolas, tal como a termografia infravermelha (IRT, infrared thermography, em inglês), permitem a captura de imagens detectando a temperatura do copado com alta resolução espacial e temporal. Esta temperatura é afectada pelo stresse hídrico o qual condiciona a produção. A monitorização da cultura e do seu estado hídrico por técnicas IRT permite o diagnóstico precoce de vários problemas das culturas, com alto potencial para economizar água, reduzir a lixiviação de nutrientes e aumentar a productividade física e económica. A presente comunicação apresenta o estudo do desenvolvimento de uma aplicação para a Web para análise de imagens térmicas IRT do coberto vegetal de uma cultura, associadas a imagens de luz visível, obtidas por detecção remota de proximidade. Esta aplicação é uma ferramenta informática que permite isolar uma região de uma imagem através da sua cor e determinar a respectiva temperatura, por exemplo do copado e do solo nu, fornecendo de forma expedita informação útil à gestão da cultura, designadamente para a gestão da rega e fertilização. Faz-se a apresentação dos procedimentos informáticos adoptados, das características das imagens processadas e de um exemplo prático de aplicação com a determinação do Î́ndice de Stresse Hídrico da Cultura baseada em observações termográficas.
\end{abstract}

\section{PALAVRAS-CHAVE}

Aplicação Web, TIC em Agricultura, Termografia Infravermelha, Índice de Stresse Hídrico da Cultura

\section{INTRODUÇÃO}

A aplicação da Internet e das tecnologias de informação em agricultura tem tido, na última década, um grande impacto em aspectos como a forma de coleta de dados, o tratamento de informação e o desenvolvimento tecnológico. A Internet na agricultura constitui uma via de disponibilização de aplicações informáticas e de modelos de simulação que permite um largo universo de utilizadores, principalmente técnicos agrícolas, contribuindo assim para a melhoria dos procedimentos e práticas neste domínio. As aplicações mais específicas nos sistemas agrícolas de regadio referem-se à gestão da rega, ao uso da sensores e de detecção remota na coleta de informação espacial e ao uso integrado da informação em controladores automáticos e inteligentes (Khanal et al, 2017; Weltzien, 2016). O consumo de água em agricultura está sob pressão da sociedade para melhorar a sua eficiência de uso, melhorando a sua produtividade e diminuindo perdas e desperdícios. Tal melhoria requer práticas adequadas de monitorização de culturas e práticas de rega eficientes. Os métodos tradicionais usados para detectar o status hídrico das culturas eram baseados na amostragem de plantas no campo; estes métodos são destrutivos, trabalhosos, sujeitos a erros significativos e têm adaptabilidade limitada, por não ser viável colher amostras em áreas grandes, nem serem facilmente automatizados. As técnicas de deteç̧ão remota para monitorização das culturas abriram novas soluções para otimizar a gestão das culturas e da água, visando melhorar a productividade da terra e da água e o rendimento do agricultor. Uma dessas técnicas é a termografia infravermelha, que detecta o estado da água da colheita medindo remotamente a temperatura do copado (Zia et al., 2013; Ishimwe et al., 2014; Mangus et al., 2016; Mangus, 2017). 
Esta comunicação apresenta o estudo do desenvolvimento de uma aplicação para a Web para análise de imagens térmicas de infravermelhos do coberto vegetal de uma cultura, associadas a imagens de luz visível, obtidas por detecção remota de proximidade. Esta aplicação é uma ferramenta informática que permite isolar uma região de uma imagem através da sua cor, em especial da folhagem verde, e a determinação da respectiva temperatura, por exemplo do copado e do solo nu, fornecendo de forma expedita informação útil à gestão da cultura, designadamente para a gestão da rega e fertilização. Faz-se a apresentação dos procedimentos informáticos adoptados, das características das imagens processadas e de um exemplo prático de aplicação de campo, onde se utilizou este software.

\section{METODOLOGIAS}

Distinguem-se aqui a metodologia utilizada no desenvolvimento da aplicação e a metodologia utilizada na experiência na qual a aplicação foi utilizada.

\subsection{Desenvolvimento da Aplicação}

\subsubsection{Objectivo}

Esta aplicação foi desenvolvida com o objectivo de facilitar e agilizar o processo de análise de dados térmicos obtidos por detecção remota de proximidade, no âmbito de trabalhos experimentais na área da agricultura. Nomeadamente, de imagens térmicas recolhidas de plantas de folhagem verde. A aplicação será utilizada, preferencialmente, por engenheiros agrónomos e por técnicos agrícolas.

Pretende-se que a aplicação leia e interprete imagens obtidas de câmaras térmicas FLIR. Para cada imagem de cor natural, o programa deve isolar, de forma fácil, rápida e consistente, uma zona que corresponda ao verde das folhas de uma planta. Havendo isolado essa região, deve ir buscar a informação da temperatura associada e realizar cálculos, apresentando-os ao utilizador.

\subsubsection{Especificações}

Esta aplicação permite o carregamento de ficheiros gerados pela FLIR C3. Estes ficheiros são compostos por três partes: informação térmica, que é a informação captada pelo sensor térmico que corresponde ao conjunto dos valores medidos pelo conversor analógico/digital da radiação recebida em cada píxel, uma imagem de luz visível e metadados, que incluem os dados de calibração de fábrica da câmara para o cálculo da temperatura. A partir da informação térmica pode ser gerada uma imagem térmica, que é uma imagem de menor resolução e contida na imagem de luz visível, pelo que é necessário proceder ao cálculo das temperaturas e ao alinhamento das mesmas.

O programa funciona em duas partes: uma parte de preparação da imagem, que inclui o carregamento e a preparação dos dados e uma parte de análise da imagem, que inclui o isolamento da região de interesse e os cálculos com as temperaturas nessa zona.

É na fase de preparação dos dados que se realiza o cálculo das temperaturas, dado pela lei de Planck e pelos valores presentes nos metadados e o alinhamento das imagens referidos anteriormente.

$\mathrm{O}$ isolamento da região de interesse é feito pela selecção de píxeis que cumpram $\mathrm{H}_{\text {corl }}<\mathrm{H}_{\text {pixel }}<\mathrm{H}_{\text {cor2 }}, \mathrm{S}_{\text {cor1 }}$

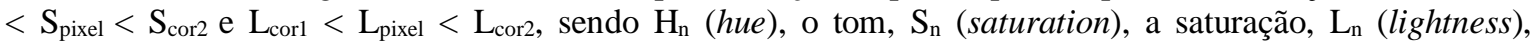
a luminosidade, corl e cor2 cores de corte, no espaço de cores HSL (Hue Saturation Lightness, em inglês), e pixel o píxel a analisar. São isolados também os píxeis da imagem térmica que correspondem aos píxeis da imagem de luz visível seleccionados. Estes passam posteriormente pelo corte de valores extremos, que consiste em retirar uma percentagem de píxeis de valores de temperatura máximos e mínimos.

Os parâmetros utilizados no isolamento da região estão pré-definidos no programa, podendo ser alterados pelo utilizador, servindo para este ajustar a área de interesse. Com os valores de temperatura dos pontos seleccionados, o programa realiza cálculos numéricos que são mostrados ao utilizador. 


\subsection{Contexto Experimental}

O trabalho experimental de campo foi desenvolvido no Aproveitamento Hidroagrícola do Baixo Mondego, numa parcela privada, cultivada com milho-grão, regado pelo método dos sulcos. Os detalhes agronómicos deste estudo estão explanados em Gonçalves et al. (2018). Foi usada uma câmara térmica portátil FLIR C3, com resolução de imagem térmica de 80 × 60 píxeis com uma precisão de cerca de $0,1^{\circ} \mathrm{C}$, colocada manualmente sobre o topo do copado, cerca de 1,5 m acima das folhas. As imagens térmicas foram obtidas semanalmente, sob condições de céu claro, próximo ao meio-dia solar.

Para avaliação da condições de conforto da cultura e a sua relação com a distribuição da água de rega na parcela, foi considerado o Índice de Stresse Hídrico da Cultura (CWSI) calculado através da equação (Jackson et al, 1981): CWSI $=\left(\mathrm{T}_{\text {copado }}-\mathrm{T}_{\text {hum }}\right) /\left(\mathrm{T}_{\text {seco }}-\mathrm{T}_{\text {hum }}\right)$, sendo $\mathrm{T}_{\text {copado }}$ a temperatura atual do copado obtida pela câmara térmica; $\mathrm{T}_{\text {hum }}$ a temperatura da folha em situação de pleno conforto hídrico, garantindo essa situação

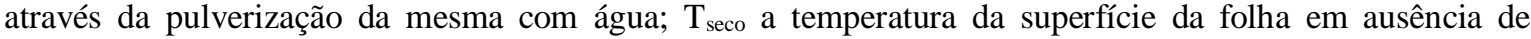
transpiração, causada por intenso stress hídrico, para o que se utilizou uma superfície de tecido verde orientada para sul. CSWI permite uma estimativa do estado de stress da cultura, compreendido entre 0 , sem stresse, e 1, máximo stress (Taghvaeian et al, 2012). Estas temperaturas $\mathrm{T}_{\text {copado, }} \mathrm{T}_{\text {hum }} \mathrm{eT}_{\text {seco }}$ são medidos no campo pela câmara térmica, nas diversas condições referidas.

\section{RESULTADOS}

\subsection{A Aplicação}

As imagens são analisadas pelo programa de forma individual e independente. Ao utilizador é permitido alterar alguns parâmetros em relação aos valores pré-definidos, como os valores para definição da cor a ser isolada e do corte dos valores extremos, sendo que, por omissão, estes valores já estão definidos.

A Figura 1 apresenta um exemplo do aspecto do programa e dos dados que ele apresenta.

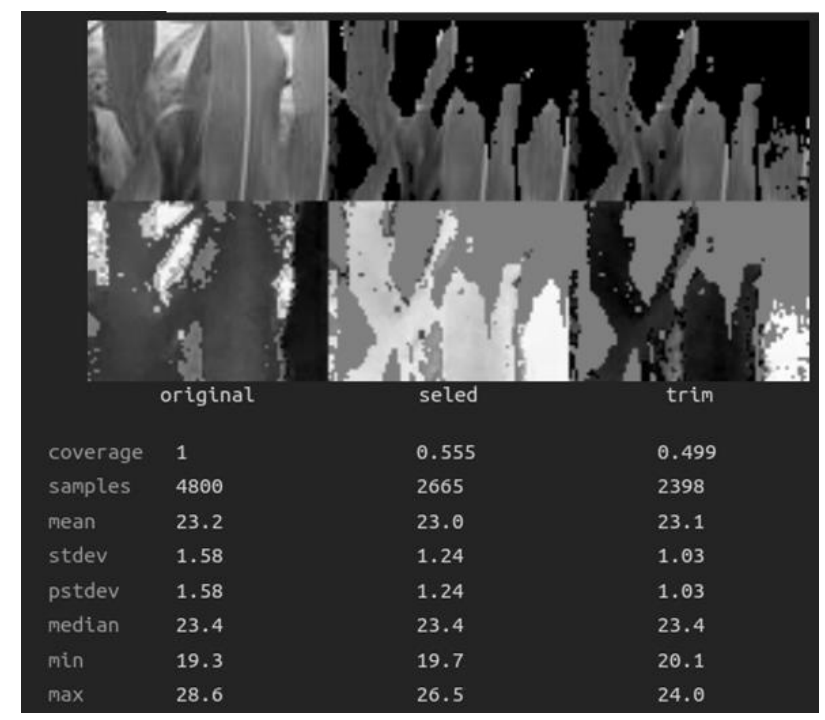

Figura 1. Exemplo de layout do programa

São mostrados três conjuntos de imagens emparelhadas de imagem de luz visível e imagem térmica, conforme exemplificado na Fig. 1. As imagens de luz visível estão redimensionadas para a região coincidente com as imagens térmicas. O primeiro par, (original) à esquerda, mostra a imagem original. O do meio, (seled), a região com a cor de interesse seleccionada. $\mathrm{O}$ da direita, (trim), a região com a cor de interesse, sem os pontos com temperaturas extremas. Os píxeis excluídos na imagem de luz visível aparecem pintados a preto e na imagem térmica, a cinzento. 
Associado a cada par de imagens aparece a análise numérica. $\mathrm{O}$ rácio de píxeis utilizado para o cálculo (coverage); o número de píxeis seleccionados (samples); a média do seu valor (mean); o desvio padrão associado, baseado na amostra (stdev) ou na população (pstdev); o valor mediano (median), o valor mínimo (min) e máximo (max).

O valor a ser utilizado pelo utilizador para o cálculo do CWSI é a média (mean) e o desvio padrão (stdev), que representa a região de interesse na imagem fotografada. Os restantes campos servem para avaliação da qualidade da selecção dos pontos e, portanto, da qualidade desse valor de interesse.

\subsection{Avaliação}

Uma vez que a aplicação está num ciclo inicial de desenvolvimento, não foram realizados testes de usabilidade formais, sendo apenas requerido aos utilizadores uma apreciação e uma crítica da utilização do programa. Os utilizadores referiram a aplicação como sendo de fácil utilização e útil para o seu propósito, tendo permitido a extracção de dados viáveis para o estudo em que se inseriam. Foram, no entanto, notadas algumas dificuldades, nomeadamente a dificuldade em ajustar os parâmetros de selecção da área de interesse. Isto explica-se tanto pela complexidade do ajuste, dado o elevado número de variáveis, como pela morosidade do processo, que tem de ser repetido para cada imagem individual.

Foram indicadas algumas sugestões de melhoramento, como a leitura de várias imagens em simultâneo, a integração de informação espacial e temporal, ou seja, do ponto no terreno a que respeita uma dada imagem, bem como a que fase de desenvolvimento da planta se refere. Isto permitiria uma análise mais rápida dos dados de todo o terreno e da sua evolução temporal. Foi também sugerida a extracção de dados gráficos. Aliada ao melhoramento anteriormente referido, permitiria a visualização gráfica do estado de todo o terreno de forma imediata. Outra sugestão feita foi a incorporação do cálculo do CWSI na aplicação.

\subsection{Aplicação dos Dados}

Apresentam-se resultados de um estudo experimental de campo, em que se efetuaram medições térmicas do copado de uma cultura de milho. A captura das imagens foi efectuada manualmente, sendo usada uma haste para colocação da câmara a uma altura suficientemente elevada para a câmara se posicionar cerca de 1 metro acima do copado. No gráfico da esquerda da Fig. 2 apresentam-se valores de CWSI em quatro dias diferentes em diversos pontos, localizados a diferentes distâncias da cabeceira da parcela, para obter representatividade espacial na parcela. Em face da natureza do sistema de rega por sulcos, à medida que aumenta esta distância ficam registados valores acrescidos de stress hídrico, a partir de maiores valores de temperatura do copado.
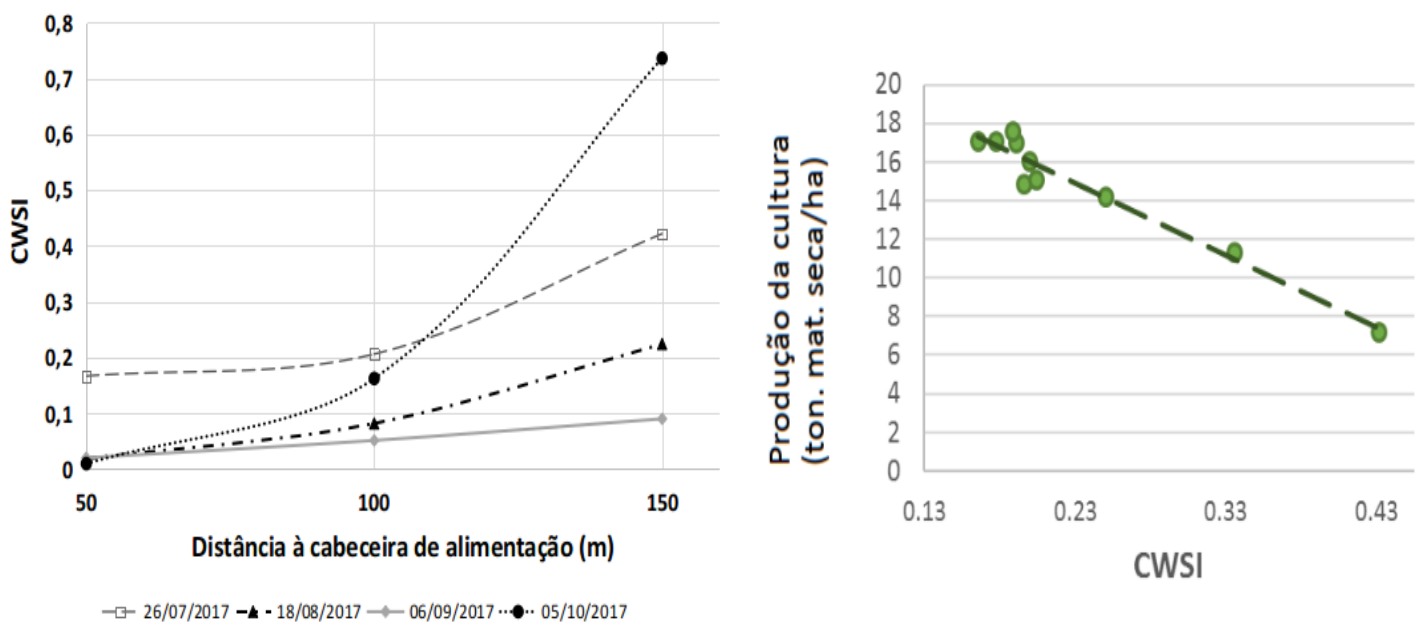

Figura 2. Exemplo de resultados do índice de stress hídrico da cultura do milho (CWSI) obtido por medições térmicas (esquerda) e a sua relação com a produtividade (direita) 
No gráfico do lado direito desta figura, apresenta-se a relação da produtividade da cultura (milho-grão seco) com os valores médios sazonais dos valores de CWSI, nos referidos pontos da cultura na parcela.

\section{CONCLUSÕES}

A aplicação para a Web destinada a analisar imagens termográficas do copado duma cultura mostrou ser uma ferramenta informática eficiente e flexível, permitindo o carregamento das imagens obtidas pela câmara térmica de forma simples, o seu registo e a determinação do valor da temperatura do copado de forma automática. No contexto agronómico da experimentação, a termografia por infravermelhos aplicada ao copado permitiu, através da facilidade em obter os dados para o cálculo do CWSI, avaliar a variabilidade do desenvolvimento da cultura na parcela. O processo de captura das imagens é melhorado se for utilizado um veículo aéreo não tripulado (drone), sendo cada imagem devidamente georeferenciada.

A aplicação poderá ser melhorada, permitindo a análise de várias imagens em simultâneo, possibilitando a extração de dados com referência espaciais e temporais, bem como a sua visualização gráfica, e o cálculo integrado do CWSI. Esta melhoria será determinante aquando da disponibilização das imagens por drone, sendo necessário o devido registo das imagens em base de dados. Outra melhoria possível é o afinamento da área de interesse a analisar de forma automática. Este último coloca-se como um desafio, dada a complexidade do problema. Há vários aspectos a considerar, desde a espécie da planta, à fase de desenvolvimento da mesma ou até mesmo à estrutura fotografada, que alteram o tom de verde de interesse na imagem, até questões mais técnicas como sombras ou regiões de alto brilho na imagem ou as limitações do sensor da câmara.

\section{AGRADECIMENTO}

i) Instituto Politécnico de Coimbra, Escola Superior Agrária de Coimbra - ESAC, Portugal; ii) Projecto HIRT - Modelling surface hydrologic processes based on infrared thermography at local and field scales (PTDC/ECM-HID/4259/2014 - POCI-01-0145-FEDER-016668); iii) Projecto NDYB2016-23, National Thirteenth Project Five-Year Scientific and Technical Support Plan, Inner Mongolia Agricultural University, China.

\section{REFERÊNCIAS}

Gonçalves, J.M. et al, 2018. Using Infrared Thermography to estimate the CWSI and furrow irrigation performance in a maize field in the Lower Mondego Valley, Portugal. Proceedings XIX World Congress of CIGR "Sustainable Life for Children", Antalya, Turkey.

Ishimwe, R. et al, 2014. Applications of Thermal Imaging in Agriculture-A Review. Advances in Remote Sensing, 3, pp. 128-140. http://dx.doi.org/10.4236/ars.2014.33011.

Jackson, R.D. et al, 1981. Canopy temperature as a crop water stress index. Water Resour. Res. 17, pp. 1133-1138.

Khanal, S. et al, 2017. An overview of current and potential applications of thermal remote sensing in precision agriculture. Computers and Electronics in Agriculture 139, 22-32.

Mangus, D.L. et al, 2016. Development and evaluation of thermal infrared imaging system for high spatial and temporal resolution crop water stress monitoring of corn within a greenhouse. Computers and Electronics in Agriculture 121, pp. 149-159.

Mangus, D.L., 2017. The basics of thermal imaging with unmanned aerial systems. Resource, March/April, pp. 16-18, ASABE, Michigan.

Taghvaeian, S. et al, 2012. Infrared Thermometry to Estimate Crop Water Stress Index and Water Use of Irrigated Maize in Northeastern Colorado. Remote Sens. 2012, 4, pp. 3619-3637. doi:10.3390/rs4113619.

Weltzien, C., 2016. Digital agriculture - or why agriculture 4.0 still offers only modest returns. LANDTECHNIK 71(2), pp. 66-68.

$\mathrm{Zia}$, S. et al., 2013. Infrared thermal imaging as a rapid tool for identifying water-stress tolerant maize genotypes of different phenology. J. Agron. Crop Sci., pp. 75-84. 